\title{
Searches for charged lepton flavor violating muon decay, MEG/MEG II experiment
}

\section{Toshiyuki Iwamoto*t}

ICEPP, the University of Tokyo

7-3-1 Hongo Bunkyo-ku, Tokyo, 113-0033, Japan

E-mail: iwamotodicepp.s.u-tokyo.ac.jp

In order to search for charged lepton flavor violating muon decay $\mu^{+} \rightarrow e^{+} \gamma$, the MEG experiment started data taking in 2009, and finished in 2013. After the careful data analysis has been performed, it is found that the result was consistent with null signal, and the upper limit of the branching ratio of the $\mu^{+} \rightarrow e^{+} \gamma$ was set to be $4.2 \times 10^{-13}$ at $90 \%$ CL while the final sensitivity was $5.3 \times 10^{-13}$. This result is the most stringent to date, about 30 times better than the previous experiment, and provides important constraints on the existence of new physics beyond the standard model. The MEG II experiment will improve the sensitivity by about an order of magnitude with higher muon beam rate and new detector technologies. The target sensitivity is $6 \times 10^{-14}$ with three years data taking. The detector R\&D has been performed seriously since the MEG experiment was finished, and the construction of each sub-detector has almost been completed. A muon beam test will be carried out for each sub-detector with limited electronics this autumn. The engineering run followed by the physics run will start next year after all the electronics will be available.

XXIX International Symposium on Lepton Photon Interactions at High Energies - LeptonPhoton2019 August 5-10, 2019

Toronto, Canada

* Speaker.

${ }^{\dagger}$ on behalf of the MEG II collaboration 


\section{Introduction}

The purpose of the MEG/MEG II experiment is to search for charged lepton flavor violating muon decays. Flavor violation phenomena already happen in the standard model. KobayashiMaskawa matrix (CKM matrix) describes quark mixing phenomena, and Maki-Nakagawa-Sakata matrix (MNS matrix) describes neutrino oscillation phenomena. However, charged lepton flavor violation has not been observed yet, and the reason is not clear yet.

$\mu^{+} \rightarrow e^{+} \gamma$ decay search has a long history since the muons was discovered. Fig. $\square$ shows the CLFV search history with muon beams.

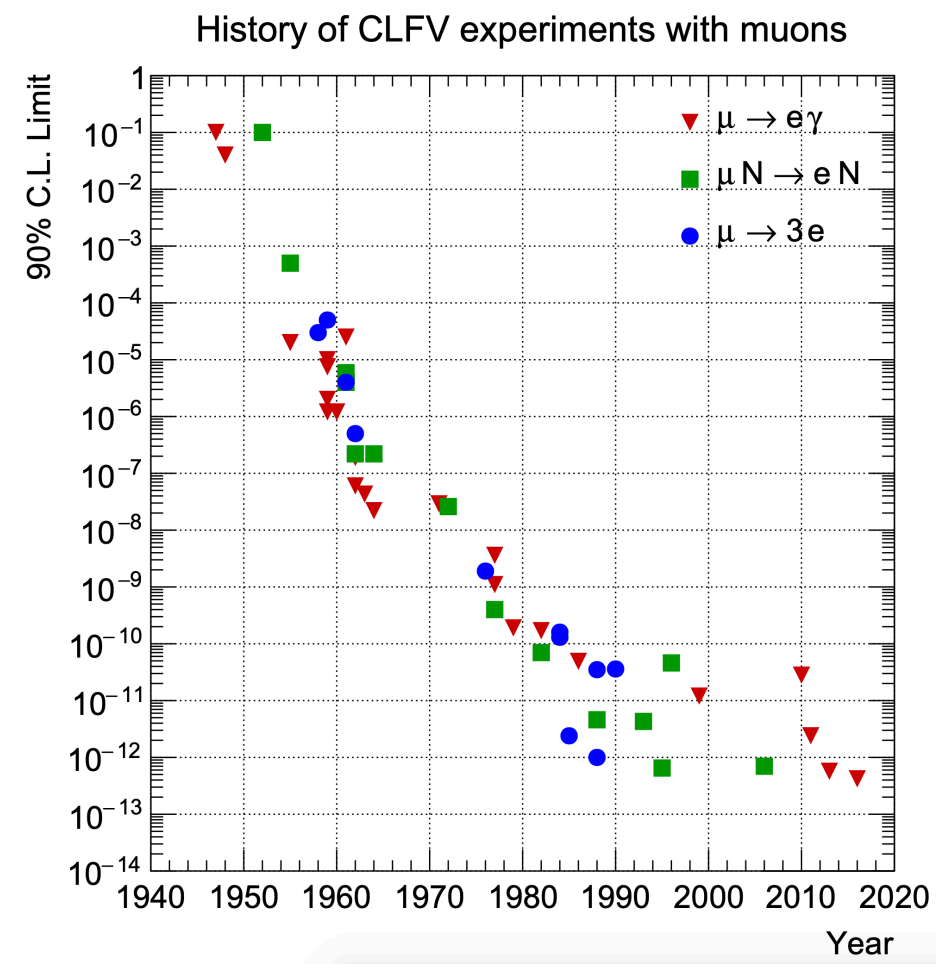

Figure 1: The charged lepton flavor violation decay search history[D]

Negative results contribute to the standard model (SM) formation. In the SM plus neutrino oscillation, the branching fraction of $\mu^{+} \rightarrow e^{+} \gamma$ is tiny, about less than $10^{-50}$, but many new physics scenarios like supersymmetric grand-unified theory (SUSY-GUT), SUSY-seesaw predict a large branching fraction through new physics in a loop diagram.

\section{MEG experiment}

The MEG experiment was designed to search for such regions where new physics like SUSYGUT, SUSY-seesaw predict. It means that there is a real chance to discover new physics. The data taking was done between 2009 and 2013. The MEG experiment set the upper limit of the branching fraction of $\mu^{+} \rightarrow e^{+} \gamma$ of $4.2 \times 10^{-13}$ at $90 \%$ C.L. [四], while the sensitivity was $5.3 \times 10^{-13}$. This is 30 times better than the previous result. The MEG collaboration prepares now the new experiment, 
the MEG II which aims at an order of magnitude better sensitivity, $6 \times 10^{-14}$ []],than the MEG experiment with three years data taking.

Other charged lepton flavor violation (CLFV) experiments are also planned to look for $\mu$-e conversion, $\mu \rightarrow 3 e$ experiment, $\tau \mathrm{LFV}$, and these results will also come soon.

The $\mu^{+} \rightarrow e^{+} \gamma$ signal and the backgrounds are as follows. The signal is simple, from the stopped muon decaying into two particles, $\gamma$ and positron. The energy is equally divided (52.8 MeV each), and the two particles are emitted back-to-back at the same time.

The dominant background source is accidentals. The usual Michel decay positron and other random $\gamma_{\mathrm{s}}$ from radiative muon decay or annihilation in flight accidentally mimic the signal. The number of accidentals is proportional to the instantaneous muon beam rate squared and the detector resolution, so the lower instantaneous muon beam rate is important and we use a DC muon beam, and better detector resolutions are important, too.

The MEG experiment was done at Paul Scherrer Institute in Switzerland, which has a $590 \mathrm{MeV}$ proton accelerator that can produce the world's most intense DC muon beam.

In the MEG experiment, the muon beam rate is reduced down to $3 \times 10^{7} \mu^{+} / \mathrm{s}$ in order to reduce the accidental background. The muon is stopped in the stopping target, and two particles are emitted isotropically. Positron's trajectory is bent by the superconducting magnet, and tracked by the segmented drift chamber. The positron time is measured by the scintillation bar timing counter. The gamma is detected by the 900L liquid xenon $\gamma$ detector with 846 PMT readout.

The MEG experiment gathered $7.5 \times 10^{14} \mu^{+}$stopped on the target in the full dataset. The five observables $\left(\mathrm{E}_{\gamma}, \mathrm{E}_{e}, \mathrm{t}_{e \gamma}, \theta_{e \gamma}, \phi_{e \gamma}\right)$ are used in the maximum likelihood analysis to extract the number of signal events, that of accidental background, and that of the radiative muon decay. The blind analysis is adopted to avoid any human bias in the parameter space of $\mathrm{E}_{\gamma}$ and $\mathrm{t}_{e \gamma}$. The probability density function for each observable is mainly extracted from the data for the accidental background, and the fitting results are well consistent with the data. Unfortunately the fit result consistent with no signal and the MEG experiment set an upper limit of the branching fraction of the $\mu^{+} \rightarrow e^{+} \gamma$ decay, $4.2 \times 10^{-13}$ at $90 \%$ C.L.

\section{MEG II experiment}

The MEG II experiment is designed to improve the MEG experiment by one order of magnitude. Fig. \ $\square$ shows the schematic view of the MEG II detector. In order to improve the experimental sensitivity, we need to increase the statistics, and the easiest way for that is to increase the muon beam intensity. In the MEG experiment, the muon beam rate was restricted to $3 \times 10^{7} \mu / \mathrm{s}$ in order to manage the number of accidentals, and the maximum beam intensity was not used. In the MEG II experiment, in order to use the PSI accelerator's maximum muon beam intensity, the resolutions of all the detector components will be improved by a factor of 2 , and the muon stopping rate at the target of $7 \times 10^{7} \mu / \mathrm{s}$ can be managed.

The drift chamber is changed from the segmented type to the single volume cylindrical shape. In the sensitive region, only the wires are distributed with small stereo angles and higher granularity, and the increased number of hits per track realize better momentum and angle resolution. The edge of the drift chamber had a readout amplifier board in the MEG experiment, which prevent the 


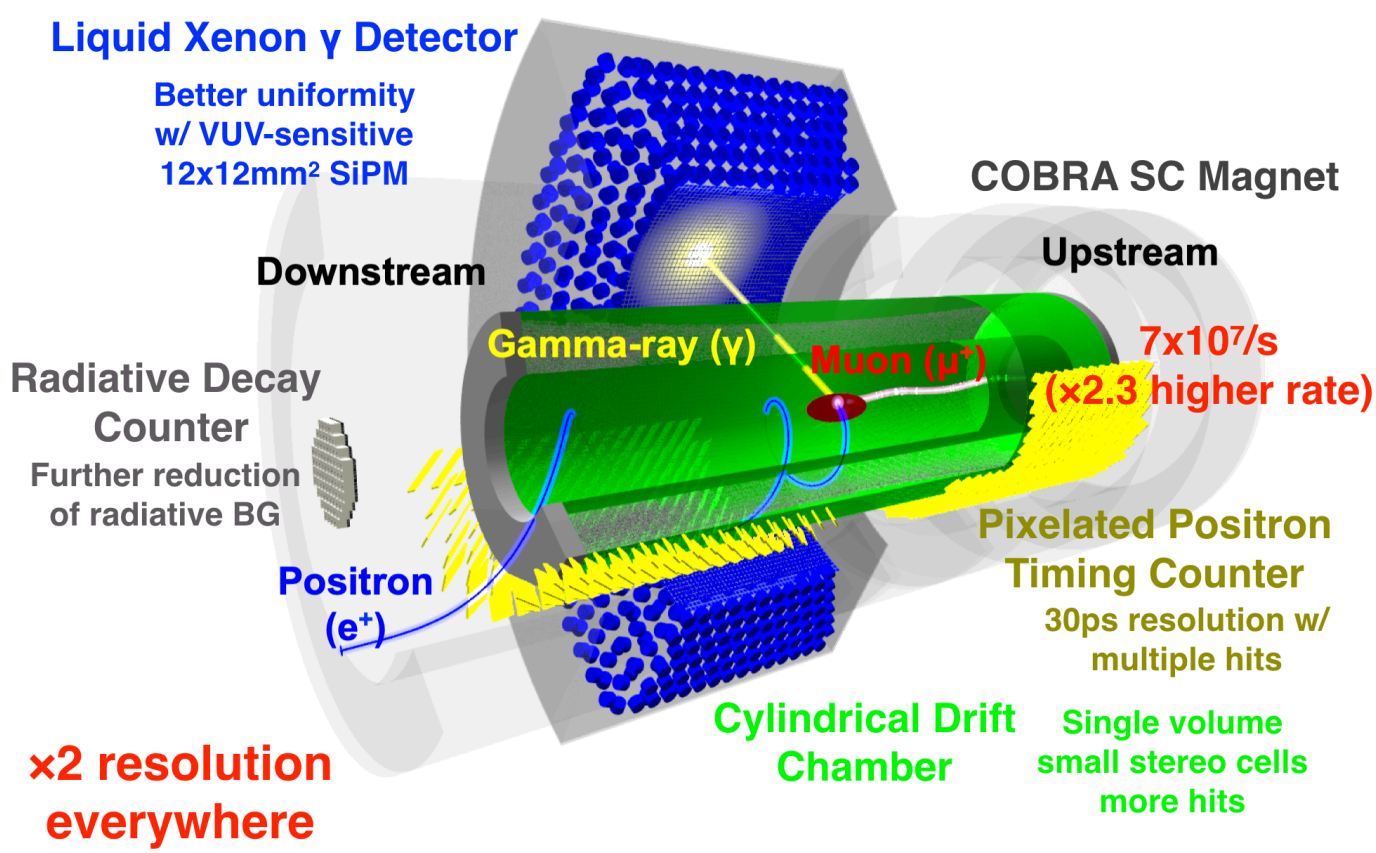

Figure 2: The MEG II detector design

positron reaching the timing counter. In the MEG II experiment, the drift chamber is now extended, and there is no extra material between timing counter and the drift chamber.

The timing counter is pixelated. 15 scintillator bars with PMTs at both ends were used in the MEG experiment for the timing counter, and 256 scintillator plates with SiPM at both ends are used in the MEG II experiment. Multiple counter hits will improve the timing resolution down to 30ps.

The liquid xenon $\gamma$ detector uses the newly developed VUV-sensitive large area MPPCs[[]] (made by Hamamatsu) on the $\gamma$ incident face. 216 PMTs were installed on the incident face, and now those are replaced with 4092 MPPCs. Better granularity, and better uniformity will improve the energy and position resolution especially for the events close to the incident face.

In order to identify the high energy background $\gamma_{\mathrm{s}}$ from the radiative muon decay, low momentum positron detection is essential. The radiative decay counter, which is made of plastic scintillator + LYSO crystal, is newly installed in the MEG II experiment to help the further reduction of the radiative muon decay background.

Waveform data are crucial for the high rate measurement. In the MEG II experiment, the number of channels are increased to realize the finer granularity, and more compact waveform digitization is necessary. The new waveform digitizer based on the DRS4 chip is developed by PSI which has many functionalities like simple trigger, bias voltage supply up to $200 \mathrm{~V}$ in a board. These are especially suitable for SiPMs. Online triggering is important to manage the high event rate and background suppression. The waveform digitizer and the FPGA based trigger system are 
integrated for the MEG II experiment, and the custom made system is begin prepared.

The sensitivity of the MEG II experiment is extracted from the ensemble of the simulated experiments (toy MC) generated from the PDFs. Data for a few months exceed the current experimental limit (set by the MEG experiment), and reach $6 \times 10^{-14}$ in three years data taking.

\section{Current status}

The construction of the drift chamber finished in 2018. The detector commissioning was carefully done last year, and will be continued this year. The construction of the timing counter finished in 2017. The basic performance test with muon beam had been performed, and the 30-40ps resolutions are already achieved. The construction of the liquid xenon detector was also finished in 2017. Since then, performance checks are ongoing, and this year, $55 \mathrm{MeV} \gamma$ from the $\pi^{0}$ decay will be used to evaluate the energy, timing, and position resolutions. The downstream radiative decay counter was constructed in 2017, and the performance test was finished with muon beam. The upstream radiative decay counter $R \& D$ is continuing. This is more difficult than the downstream side because the muon beam will penetrate the counter in the upstream side. Only a limited number of readout electronics channels $(\sim 1500 \mathrm{ch}$.) are currently available, and these are being tested by sub-detectors. The mass production of the electronics will be carried out next year.

\section{Summary}

Currently the MEG II detector integration is almost finished, and muon beam time started in September 2019 with a limited number of electronics channels. The mass production of the readout electronics happens next year. The sensitivity of the MEG II experiment will exceed the current limit with a few months of data, and will improve by one order of magnitude with three years data taking.

\section{Acknowledgements}

This work is supported by MEXT/JSPS KAKENHI Grant Numbers JP22000004, JP26000004, and JSPS Core-to-Core Program, A. Advanced Research Networks.

\section{References}

[1] A. M. Baldini et al. [MEG Collaboration], Eur. Phys. J. C 76 (2016) no. 8,434 doi:10.1140/epjc/s10052-016-4271-x [arXiv:1605.05081 [hep-ex]].

[2] A. M. Baldini et al. [MEG II Collaboration], Eur. Phys. J. C 78, no. 5, 380 (2018) doi:10.1140/epjc/s10052-018-5845-6 [arXiv:1801.04688 [physics.ins-det]].

[3] W. Ootani et al., Nucl. Instrum. Meth. A 787, 220 (2015). doi:10.1016/j.nima.2014.12.007 